\title{
O método Lee Silverman para reabilitaçáo da fala na doença de Parkinson
}

\author{
The Lee Silverman method for rehabilitation of speech in Parkinson's disease
}

Alice Estevo Dias', Hsin Fen Chien², Egberto Reis Barbosa ${ }^{3}$

\begin{abstract}
RESUMO
As alteraçôes da fala (disfonia e disartria) frequentemente acompanham a evoluçáo da doença de Parkinson (DP). Objetivo. Este estudo revisa o Método Lee Silverman, considerado o mais eficiente para a reabilitação das alteraçôes da fala na DP e atualiza os avanços na sua aplicação. Método. Foi realizada uma pesquisa nas bases de dados MEDLINE, Pubmed e Bireme dos artigos indexados publicados de 1990 a 2010, com as seguintes palavras-chave: Parkinson's disease, PD, Lee Silverman Voice Treatment, LSVT, LSVT LOUD, LSVT parkinson, voice treatment and $P D$, voice therapy and $P D$, communication and $P D$, dysarthria and PD, dysphonia and PD, speech disorders and PD, voice disorders and $P D$, hypophonia and $P D$, speech motor system and $P D$. Resultados. Na literatura, existe ampla descrição dos resultados de estudos do método Lee Silverman na DP. Os artigos encontrados evidenciam melhora da prosódia, articulação, ressonância, respiração, inteligibilidade, intensidade e qualidade da voz, assim como da deglutição e da expressividade facial. Conclusóes. Para a reabilitação da fala dispóe-se de eficientes técnicas fonoaudiológicas. Contudo, conforme a literatura científica, o emprego do Método Lee Silverman é opção vantajosa, pois foi desenvolvido especificamente para a DP. Há inúmeras evidências de sua eficácia e vem sendo continuamente avaliado, ampliando sua aplicabilidade.
\end{abstract}

Unitermos. Doença de Parkinson, Fala, Voz, Reabilitação.

Citaçáo. Dias AE, Chien HF, Barbosa ER. O método Lee Silverman para reabilitação da fala na doença de Parkinson.
Trabalho realizado no Ambulatório de Distúrbios do Movimento da Divisáo de Neurologia do Hospital das Clínicas da Faculdade de Medicina da Universidade de Sáo Paulo, São Paulo-SP, Brasil.

1. Fonoaudióloga mestre e doutora em Ciências pela Faculdade de Medicina da Universidade de Sáo Paulo (FMUSP) e membro do Grupo de Estudo de Distúrbios do Movimento da Divisão de Neurologia do Hospital das Clínicas da FMUSP, São Paulo-SP, Brasil.

2. Neurologista, mestre e doutora pela FMUSP e médica colaboradora do Grupo de Estudo de Distúrbios do Movimento da Divisáo de Neurologia do Hospital das Clínicas da FMUSP, São Paulo-SP, Brasil.

3. Neurologista, Livre-Docente do Departamento de Neurologia da FMUSP e Coordenador do Grupo de Estudo de Distúrbios do Movimento da Divisão de Neurologia do Hospital das Clínicas da FMUSP, São Paulo-SP, Brasil.

\begin{abstract}
Speech impairment (dysphonia and dysarthria) is a frequent manifestation in the evolution of Parkinson's disease (PD). Objective. This study reviews the Lee Silverman Method, considered the most efficient for the rehabilitation of PD speech impairment, and updates its most recent apllications. Method. We search the following data base: MEDLINE, Pubmed e Bireme using the words: Parkinson's disease, PD, Lee Silverman Voice Treatment, LSVT, LSVT LOUD, LSVT parkinson, voice treatment and PD, voice therapy and PD, communication and $\mathrm{PD}$, dysarthria and $\mathrm{PD}$, dysphonia and $\mathrm{PD}$, speech disorders and PD, voice disorders and PD, hypophonia and PD, speech motor system and PD. Results. In the literature, there are many descriptions of good results of the Lee Silverman method in PD. The articles depict improvement in the following topic: prosody, articulation, ressonance, respiration, intelligibility, intensity, and quality of voice, as well swallowing and facial expressiveness. Conclusion. There are many efficient speech therapy techniques for voice rehabilitation. But, according to the literature, the Lee Silverman Method is advantageous since it was develop specifically for PD. There are innumerable evidences for its efficacy, moreover it has been constantly evaluated and its application is broaden continually.
\end{abstract}

Keywords. Parkinson's Disease, Speech, Voice, Rehabilitation.

Citation. Dias AE, Chien HF, Barbosa ER. The Lee Silverman method for rehabilitation of speech in Parkinson's disease.
Endereço para correspondência: Alice E Dias Rua Ouvidor Peleja 235/131 CEP 04128-000, São Paulo-SP, Brasil. E-mail: dialice@terra.com.br 


\section{INTRODUÇÃO}

\section{A doença de Parkinson e a fala}

A doença de Parkinson (DP) é uma condição que representa até $2 / 3$ dos pacientes que procuram as clínicas especializadas em Distúrbios do Movimento e é classificada como a segunda mais prevalente dentre as doenças neurodegenerativas ${ }^{1}$. No Brasil, a prevalência é estimada em $0.3 \%$ da população geral e afeta $3.3 \%$ dos indivíduos com mais de 65 anos de idade 2 .

A DP é descrita como uma enfermidade degenerativa do sistema nervoso central (SNC), de evolução inexorável, caracterizada pela perda progressiva de neurônios em diversas regiôes do SNC e especialmente da parte compacta da substância negra do mesencéfalo. $\mathrm{Na}$ maioria dos casos as manifestaçóes clínicas surgem entre a $6^{a}$ e a $7^{a}$ décadas da vida.

O diagnóstico da doença é essencialmente clínico, e baseia-se na presença de sinais e sintomas motores, representados por tremor, rigidez, bradicinesia e alteraçóes nos reflexos posturais.

A combinaçáo da rigidez e da bradicinesia associadas a prejuízos no processamento sensorial e cognitivo ${ }^{3}$, desencadeiam modificaçóes na fala (disfonia e disartria) em alguma fase da doença. Essas modificaçóes em conjunto levam a um tipo particular de alteração denominada disartria hipocinética ${ }^{4}$. Esta alteração caracteriza-se por movimentos lentos, fracos, imprecisos ou incoordenados da musculatura envolvida na produção da fala, devido a transtornos na execução motora e também no processo de planejamento da produção, que envolve múltiplas áreas do encéfalo como núcleos da base, cerebelo, área motora suplementar e circuitos frontais 5 .

Estudos apontam que até $90 \%$ das pessoas com DP podem apresentar alteraçóes da fala, de modo a apresentar anormalidades que englobam o sistema de ressonância, respiração, voz, e articulaçẫo ${ }^{6}$, interpretadas como originárias de danos no planejamento motor e dificuldades na execução de programas simultâneos ou sequenciais próprios de disfunções dos núcleos da base?

A ressonância diz respeito à habilidade de projeção da voz. Na DP, alteraçóes nesse sentido são relacionadas ao fechamento do esfíncter velo-faríngeo, à diminuição na amplitude de abertura da boca e à falta de pressão intra-oral.
A respiração é geradora da energia necessária para a emissão da voz. O processo da respiração requer controle complexo dos músculos respiratórios e este pode estar comprometido na DP em decorrência da reduzida movimentação da caixa torácica, denotando emprego insuficiente de ar durante a produção da voz e consequente diminuição da intensidade da voz, emissão de frases encurtadas, velocidade da fala variada e interrupçóes abruptas.

A produção da voz é sensivelmente prejudicada na DP em virtude de alteraçóes laríngeas que abrangem redução da mobilidade, arqueamento e fenda ânteroposterior das pregas vocais e hiperfunção supraglótica. As alteraçôes da voz encontradas caracterizam-se por insuficiência da prosódia, caracterizada por monotonia, devido às restriçóes na modulação de frequência e de intensidade, redução da intensidade (hipofonia) e alteraçóes da qualidade, normalmente rouquidão e soprosidade.

A articulação é um processo de posicionamento e movimentação das estruturas orofaciais (lábios, língua e bochechas) para a produção concatenada de segmentos fonêmicos em uma série de tempo. As alteraçóes articulatórias na DP incluem discurso hesitante/disfluente e são caracterizadas por consoantes imprecisas, que refletem as limitações na movimentação das estruturas orofaciais e os prejuízos no grau de abertura e fechamento da boca, em seus diferentes pontos de articulação.

\section{A reabilitaçáo da fala na DP}

Possivelmente as alteraçóes da fala presentes na DP resultam de lesões não-dopaminérgicas ${ }^{8}$ e diferente do tremor, da bradicinesia e da rigidez, são pouco responsivas ao tratamento com levodopa, principalmente nos estágios intermediários e avançados da doença?.

Há evidências de que os tratamentos farmacológico, cirúrgico e as tradicionais técnicas de reabilitação fonoaudiológica são pouco eficientes para a reabilitação da fala dos pacientes com $\mathrm{DP}^{10}$.

O tratamento fonoaudiológico tradicional é normalmente realizado por meses seguidos e é considerado de longa duração. A despeito do tratamento indicado para a DP englobar consagradas abordagens que envolvem técnicas e procedimentos para a coordenação das estruturas da articulação, da voz e da respiração, o mesmo não oferece resultados positivos, provavelmente por não 
considerar as peculiaridades da doença.

Um grupo de fonoaudiólogos americanos desenvolveu um programa de tratamento para as alteraçóes da fala e da voz direcionado aos pacientes com DP denominado método Lee Silverman Voice Treatment (LSVT ou LSVT LOUD). Em nosso meio, esse tratamento é conhecido como Lee Silverman, e é aplicado por fonoaudiólogos treinados e certificados internacionalmente.

O objetivo deste estudo de revisão de literatura é apresentar o método Lee Silverman, considerado o mais eficiente para a reabilitação das alterações da fala na DP e atualizar os avanços na sua aplicação.

\section{MÉTODO}

Este estudo é uma revisão de literatura sobre a doença de Parkinson e a reabilitação da fala pelo método Lee Silverman. Para tanto, a produção científica relacionada à doença de Parkinson e o método Lee Silverman foi identificada mediante a realização de um levantamento bibliográfico. Os bancos de dados inclusos para pesquisa foram MEDLINE, Pubmed e Bireme empregando a seguinte estratégia de busca: pesquisa restrita ao período de janeiro de 1990 a setembro de 2010. Para esta pesquisa foram utilizados os seguintes descritores: Parkinson's disease, PD, Lee Silverman Voice Treatment, LSVT, LSVT LOUD, LSVT parkinson, voice treatment and $P D$, voice therapy and $P D$, communication and $P D$, dysarthria and $P D$, dysphonia and $P D$, speech disorders and $P D$, voice disorders and $P D$, hypophonia and PD, speech motor system and PD. Referências citadas nas publicações relevantes foram examinadas nesta revisão, perfazendo um total de quarenta e três artigos científicos, uma dissertação de mestrado e dois trabalhos apresentados em congressos internacionais.

\section{RESULTADOS}

\section{O método Lee Silverman}

O LSVT é um método de tratamento desenvolvido para a reabilitação da fala na $\mathrm{DP}^{11}$ com nível de evidência $\mathrm{I}^{12,13}$. Em sua forma clássica, é um tratamento com duração de um mês e consta de 16 sessôes aplicadas quatro vezes por semana. Nesse período, uma sequência de exercícios é realizada de modo a automatizar o uso da voz em forte intensidade a partir do aprendizado motor, do estímulo e da motivação ${ }^{14,15}$.
Cada uma das sessóes do tratamento tem duração de uma hora e é dividida em duas partes: tarefas diárias e fala hierárquica. As tarefas diárias são aplicadas com o objetivo de aumentar a intensidade da voz por meio de múltiplas e variadas repetiçóes da vogal sustentada "a" e frases funcionais (escolhidas pelos pacientes e representativas da rotina diária). A fala hierárquica diz respeito à produção progressiva do discurso (palavras, sentenças e conversação) para que os pacientes possam treinar o aumento da intensidade da voz adquirida durante a realização das tarefas diárias e melhorar a fala funcional.

Trata-se de um programa de tratamento intensivo cujo treinamento estimula os pacientes à realizaçáo de exercícios simples e repetitivos apoiados na produção de voz forte. Diante das alteraçôes sensoriais, os pacientes são constantemente solicitados a monitorar a intensidade da voz e o esforço empregado para produzi-la, o que culmina na melhoria da propriocepção a partir da audição ${ }^{16}$.

O LSVT envolve cinco concepções essenciais que o diferencia dos demais tratamentos tradicionais: foco na voz, esforço intenso, tratamento intensivo, calibração e quantificação ${ }^{17}$.

A primeira concepção diz respeito ao foco na voz, pois a hipofonia observada na DP é associada à alteração postural das pregas vocais (fenda glótica) e ao comprometimento respiratório. $\mathrm{O}$ tratamento promove melhoria da adução das pregas vocais e do suporte respiratório que aumentam a intensidade da voz. A abordagem para o aumento da intensidade da voz é simples, pois os pacientes tem dificuldade de realizar tarefas complexas e movimentos diversos simultaneamente. Com o LSVT os pacientes não precisam de muitas instruçóes para falar bem, eles precisam memorizar apenas uma idéia: "Fale forte. Pense forte" ou "Seja forte".

A segunda concepção tem ligação com o esforço intenso de produção da voz. O método é baseado na premissa de que os pacientes, ao aumentarem o esforço muscular para falar mais forte, podem transpor a barreira da rigidez e da bradicinesia e melhorar o funcionamento da musculatura envolvida não apenas da voz, mas também da articulação, prosódia, respiração e ressonância. Essas melhoras secundárias ao alvo principal, a voz, são condizentes com os princípios da plasticidade neural ${ }^{18,19}$.

A terceira concepção baseia-se na idéia de que esta 
modalidade de tratamento promove a oportunidade diária de prática do aumento do esforço para falar forte. Isso é consistente com os princípios de aprendizado motor e aquisição de habilidades ${ }^{20}$.

A quarta concepção relaciona-se à calibração, que se refere ao emprego subjetivo da quantidade de esforço necessária para falar forte. A calibração é imprescindível para que os pacientes usem a voz forte automaticamente na comunicação diária. Para tanto, eles devem estar convencidos de que a voz precisa de grande amplitude de energia, produzida pelo esforço e dentro dos limites de conforto. Isso se faz necessário, pois os pacientes referem que estão falando muito alto, quando na verdade estão falando adequadamente. Isso se deve ao fato de que eles possuem uma interrupção entre a apreciação perceptual do objetivo da tarefa e a distribuição da instrução apropriada pelo córtex motor.

A quinta e última concepção é a quantificação, que tem como objetivo estimular o aumento do esforço para falar forte. Geralmente é realizada por meio de programas computadorizados desenvolvidos para a mensuração da fala, escalas perceptuais subjetivas do paciente, membros da família e do fonoaudiólogo ou gravaçóes em áudio e vídeo, para que os pacientes possam avaliar o desempenho, obter motivação e adquirir confiança.

\section{O método Lee Silverman de reabilitaçáo da fala na DP}

Desde o início das investigaçóes científicas, o método Lee Silverman demonstrou resultados favoráveis para a reabilitação da fala quando aplicado em grupos de pacientes com DP. O aumento da intensidade da voz e, portanto melhora da hipofonia, foi identificado em ensaios semelhantes que recorreram a metodologias distintas $^{21-25}$. Alguns estudos apontaram que o método se mostrou mais eficiente quando comparado a outros tratamentos fonoaudiológicos tradicionais. Em três estudos semelhantes, dois grupos de pacientes com DP foram avaliados do ponto de vista da fala antes e depois de tratamento. Um deles foi submetido ao tratamento pelo LSVT e o outro pelo tratamento com ênfase em exercícios respiratórios. Os resultados revelaram que a qualidade e a intensidade da voz, assim como a respiração melhoraram significativamente apenas no grupo tratado pelo LSVT ${ }^{26-28}$.
Os estudos de Ramig et al. (2001) e Sapir et al. (2002) compararam o tratamento baseado em exercícios respiratórios e o LSVT quanto aos resultados a longo prazo (1e 2 anos). Os resultados observados indicaram que a melhora obtida com o LSVT foi mais consistente e manteve-se por um período mais duradouro que a obtida com a técnica baseada na respiração ${ }^{29,30}$.

Em revisão sobre a reabilitação da fala na $\mathrm{DP}$ publicada em 2003 pela Academy of Neurologic Communication Disorders and Sciences (ANCDS) o LSVT foi considerado o tratamento mais eficaz ${ }^{31}$.

O programa de treinamento implementado pelo LSVT direcionado para o aumento da intensidade da voz conduz à modulação dos programas motores envolvidos e facilita a propagação para os outros subsistemas da comunicação verbal como articulação, respiração, prosódia e ressonância. Contudo a comunicação não verbal também foi contemplada pelo método, conforme demonstrou estudo sobre a melhora da expressividade facial (hipomimia) após o tratamento com o LSVT na $\mathrm{DP}^{32}$.

Os efeitos do LSVT na articulação foram avaliados. Os resultados obtidos a partir da análise perceptual e também acústica computadorizada da produção de vogais evidenciaram que a aplicação do Lee Silverman oferece um impacto terapêutico generalizado nas funções orofaciais da DP e que é capaz de promover significativa melhora na configuração da articulação ${ }^{33}$.

Estudos que analisaram a deglutição de pacientes com DP apontaram que as mudanças fisiológicas promovidas pela reabilitação da fala com o LSVT são capazes de desencadear transformaçóes na atividade e na sinergia da musculatura laríngea e supralaríngea, resultando em melhora da deglutição $0^{34,35}$.

Os correlatos neurais da hipofonia em pacientes com DP foram investigados antes e depois da aplicação da Lee Silverman com PET. O estudo apontou que a melhora da hipofonia foi acompanhada de redução de ativaçôes no córtex pré motor e motor, bem como do recrutamento da insula anterior direita, caudado, putâmen e córtex dorso lateral pré frontal. Esses resultados sugeriram que a diminuição da hipofonia promoveu a mudança de ativações atípicas para a emissão da voz no córtex pré motor para outras mais automáticas (núcleos da base e insula anterior) ${ }^{36}$. 
Outro estudo que envolveu PET nos períodos pré e pós tratamento pelo Lee Silverman, revelou que os satisfatórios efeitos terapêuticos na hipofonia da DP resultam do deslocamento da atividade cortical para o hemisfério direito do cérebro ${ }^{37}$.

A despeito de sua eficácia comprovada o método Lee Silverman continua sendo reavaliado e aprimorado com o intuito de melhorar sua aplicabilidade e resultados semelhantes aos alcançados com o sistema de aplicação tradicional do método tem sido obtidos. Esses avanços constam nos tópicos abaixo:

LSVT em grupo: Programa de treinamento intensivo tradicional, constituído por 16 sessóes distribuídas em quatro semanas, realizadas quatro vezes por semana e administrado em grupos de cinco pessoas ${ }^{38}$.

LSVTC: Programa de treinamento intensivo tradicional, constituído por 16 sessôes distribuídas em quatro semanas, realizadas quatro vezes por semana e administrado individualmente a partir de Personal Digital Assistant (PDA) ${ }^{39}$.

LSVTVT: Programa de treinamento intensivo tradicional, constituído por 16 sessóes distribuídas em quatro semanas, realizadas quatro vezes por semana e administrado individualmente a partir de programa computadorizado $^{40}$.

LSVT-X: Programa de treinamento intensivo tradicional, constituído por 16 sessóes distribuídas em oito semanas, realizadas duas vezes por semana e administrado individualmente ${ }^{41}$.

LSVT com videofone: Programa de treinamento intensivo tradicional, constituído por 16 sessōes distribuídas em quatro semanas, realizadas quatro vezes por semana e administrado individualmente a partir de videofone instalado na residência dos pacientes ${ }^{42}$.

LSVT pela internet: Programa de treinamento intensivo tradicional, constituído por 16 sessôes distribuídas em quatro semanas, realizadas quatro vezes por semana e administrado individualmente a partir da World Wide $\mathrm{Web}^{43}$.
LSVT por videoconferência: Programa de treinamento intensivo tradicional, constituído por 16 sessóes distribuídas em quatro semanas, realizadas quatro vezes por semana e administrado individualmente a partir de videoconferência ${ }^{44}$.

LSVT adaptado: Programa de treinamento intensivo tradicional, constituído por 16 sessóes distribuídas em dezesseis semanas, realizadas uma vez por semana e administrado individualmente ${ }^{45}$.

\section{CONCLUSÕES}

A alteração da fala na DP afeta não somente a habilidade de comunicação verbal, mas também a capacidade de interação social, profissional e familiar. Diante disso, a dificuldade e até mesmo a impossibilidade da expressão de pensamentos, desejos e idéias compromete a existência plena, acentua a insatisfação com a doença e prejudica a qualidade de vida.

Assim como ocorrem contínuos avanços na busca por uma melhor compreensão dos mecanismos envolvidos na DP e na mitigação de suas manifestações, também a fonoaudiologia se empenha na mesma direção.

Conforme exposto acima, as alterações da fala podem ser minimizadas a partir da aplicação do método Lee Silverman, que é considerado como tratamento bem sucedido na DP. Além dos benefícios primários e secundários, o método apresenta ainda, a vantagem do estabelecimento de começo e término do tratamento.

Vale ressaltar que os objetivos do tratamento são reduzir o prejuízo funcional, tornando a comunicação possível, ainda que persistam limitaçôes. O prognóstico é mais favorável quando o tratamento das alteraçóes da fala é instituído precocemente.

\section{REFERÊNCIAS}

1.Lau LM, Breteler MM. Epidemiology of Parkinson's disease. Lancet Neurol. 2006;5:525-35.

2.Barbosa MT, Caramelli P, Maia DP, Cunningham MCQ, Guerra HL, LimaCosta MF, et al. Parkinsonism and Parkinson's disease in the elderly: a community-based survey in Brazil (the Bambui study). Mov Disord 2006;21:800-8. http://dx.doi.org/10.1002/mds.20806 
3.Fox C, Halpern A, Petska J, Spielman J, Will L, Ramig L. Voice treatment $\left(\mathrm{LSVT}^{\circ}\right)$ for individuals with Parkinson disease: new horizons. Perspectives on voice and voice disorders 2005;15:9-16.

4.Darley FL, Aronson AE, Brown J. Clusters of deviant speech dimensions in the dysarthrias. J Speech Hear Res 1969;12:462-9.

5.Spencer KA, Rogers MA. Speech motor programming in hypokinetic and ataxic dysarthria. Brain 2005;94:347-66.

6.Ciucci MR, Ma ST, Fox C, Kane JR, Ramig LO, Schallert R. Qualitative changes in ultrasonic vocalization in rats after unilateral dopamine depletion or haloperidol: a preliminary study. Behav Brain Res 2007;182:284-9.

http://dx.doi.org/10.1016/j.bbr.2007.02.020

7.Widgruber D, Riecker A, Hertrich I, Erb M, Grodd W, Ethofer T, et al. Identification of emotional intonation evaluated by fMRI. Neuroimage 2005;24:1233-41.

http://dx.doi.org/10.1016/j.neuroimage.2004.10.034

8.Levy YS, Gilgun-Sherki Y, Melamed E, Offen D. Therapeutic potential of neurotrophic factors in neurodegenerative diseases. BioDrugs 2005;19:97-127. http://dx.doi.org/10.2165/00063030-200519020-00003

9.Plowman-Prine EK, Okun MS, Sapienza CM, Shrivastav R, Fernandez HH, Foote KD, et al. Perceptual characteristics of Parkinsonian speech: a comparison of the pharmacological effects of levodopa across speech and non-speech motor systems. NeuroRehabilitation 2009;24:131-44.

10.Agid Y, Graybiel AM, Ruberg M, Hirsch E, Blin J, Dubois B, et al. The efficacy of levodopa treatment declines in the course of Parkinson's disease: do nondopaminergic lesions play a role? Adv Neurol 1990;53:83-100.

11.Fox CM, Morrison CE, Ramig LO, Sapir S. Current perspectivies on the Lee Silverman Voice Treatment (LSVT) for people with idiopathic Parkison's disease. Am J Speech Lang Pathol 2002;11:111-23.

http://dx.doi.org/10.1044/1058-0360(2002/012)

12.Pinto S, Ozsancak C, Tripoliti E, Thobois S, Limousin-Dowsey P, Auzou P. Treatments for dysarthria in Parkinson's disease. Lancet 2004;3:547-56.

http://dx.doi.org/10.1016/S1474-4422(04)00854-3

13.Trail M, Fox C, Ramig L, Sapir S, Howard J, Lai EC. Speech treatment for people with Parkinson's disease. NeuroRehabilitation 2005;20:205-21.

14.Fox CM, Ramig LO, Ciucci MR, Sapir S, McFarland DH, Farley BG. The science and practice of LSVT/LOUD: neural plasticity-principled approach to treating individuals with Parkinson disease and other neurological disorders. Semin Speech Lang 2006;27:283-99.

http://dx.doi.org/10.1055/s-2006-955118

15.Sapir S, Spielman JL, Ramig LO, Story BH, Fox C. Effects of intensive voice treatment (the Lee Silverman Voice Treatment [LSVT]) on vowel articulation in dysarthric individuals with idiopathic Parkinson disease: acoustic and perceptual findings. J Speech Lang Hear Res 2007;50:899-912.

http://dx.doi.org/10.1044/1092-4388(2007/064)

16.Skodda S, Schlegel U. Speech rate and rhythm in Parkinson's disease. Mov Disord 2008;23:985-92.

http://dx.doi.org/10.1002/mds.21996

17.Ramig LO, Fox C, Sapir S. Speech treatment for Parkinson's disease. Expert Rev Neurother 2008;8:297-309.

http://dx.doi.org/10.1586/14737175.8.2.297

18.Narayana S, Fox PT, Zhang W, Franklin C, Robin DA, Vogel D, et al. Neural correlates of efficacy of voice therapy in Parkinson's disease identified by performance-correlation analysis. Hum Brain Mapp 2010;31:222-36.

19.Liotti M, Ramig LO, Vogel D, New P, Cook CI, Inghan RJ, et al. Hypophonia in Parkinson's disease: neural correlates of voice treatment revealed by PET. Neurology 2003;60:432-40.

20.Klein JA, Jones TA. Principles of experience-dependent neural plasticity: Implications for rehabilitation after brain damage. J Speech Lang Hear Res
2008;51:S225-S39.

http://dx.doi.org/10.1044/1092-4388(2008/018)

21.Ramig L, Bonitati CM, Lemke JH, Horii Y. Voice treatment for patients with Parkinson disease: development of an approach and preliminary efficacy data. J Med SpeechLang Pathol 1994;2:191-209.

22.Smith M, Ramig L, Dromey C, Perez K, Samandari R. Intensive voice treatment in Parkinson's disease: laryngostroboscopic findings. J Voice 1995;9:453-9.

http://dx.doi.org/10.1016/S0892-1997(05)80210-3

23.Ramig LO, Countryman S, Thompson L. Efficacy of voice therapy for patientes with Parkinson's disease. J Speech Hear Res 1995;97:164-72.

24.Ramig LO, Sapir S, Fox C, Countryman S. Changes in vocal following intensive voice treatment (LSVT $\left.{ }^{\circ}\right)$ in individuals with Parkinson's disease: a comparison with untreated patientes and normal age-matched controls. Mov Disord 2001;16:79-93.

http://dx.doi.org/10.1002/1531-8257(200101)16:1<79::AIDMDS1013>3.0.CO;2-H

25.Dias AE, Limongi JCP. Treatment of vocal symptoms in Parkinson's disease: the Lee Silverman method. Arq Neuropsiquiatr 2003;61:61-6.

http://dx.doi.org/10.1590/S0004-282X2003000100011

26.Ramig L, Countryman S, Thompson L, Horii Y. A comparison of two forms of intensive speech treatment for Parkinson disease. J Speech and Hear Res 1995;38:1232-51.

27.Ramig LO, Countryman S, O’Brien C, Hoehn M, Thompson L. Intensive speech treatment for patients with Parkinson's disease: short and long-term comparison of two techniques. Neurology 1996;47:1496-504.

28. Baumgartner C, Sapir S, Ramig L. Voice quality changes following phonatory- respiratory effort Treatment (LSVT) versus respiratory effort treatment for individuals with Parkinson disease. J Voice 2001;15:105-14.

http://dx.doi.org/10.1016/S0892-1997(01)00010-8

29.Sapir S, Ramig L, Hoyt P, O’Brien C, Hoehn M. Speech loudness and quality 12 months after intensive voice treatment (LSVT) for Parkinson's disease: a comparison with an alternative speech treatment. Folia Phoniatr 2002;54:296303.

http://dx.doi.org/10.1159/000066148

30.Ramig LO, Sapir S, Countryman S, Pawlas AA, O’Brien C, Hoehn M, et al. Intensive voice treatment (LSVT ${ }^{\circ}$ ) for patients with Parkinson's disease: a 2 year follow up. J Neurol Neurosurg Psychiatry 2001;71:493-98.

http://dx.doi.org/10.1136/jnnp.71.4.493

31.Yorkston KM, Spencer KA, Duffy JR. Behavioral management of respiratory/phonatory dysfunction from dysarthria: a systematic review of the evidence (ANCDS Bulletin Board). J Med Speech Lang Pathol 2003;11(2):xxxix-lxi. 32.Spielman JL, Borod JC, Ramig LO. The effects of intensive voice treatment on facial expressiveness in Parkinson disease: preliminary data. Cogn Behav Neurol 2003;16:177-88.

http://dx.doi.org/10.1097/00146965-200309000-00005

33.Sapir S, Spielman J, Ramig L, Story B, Fox C. Effects of intensive voice treatment (LSVT) on vowel articulation in dysarthric individuals with idiopathic Parkinson's disease: Acoustic and perceptual findings. J Speech Lang Hear Res 2007;50:899-912.

http://dx.doi.org/10.1044/1092-4388(2007/064)

34.El Sharkawi A, Ramig LO, Logemann JA, Pauloski BR, Rademaker AW, Smith $\mathrm{CH}$, et al. Swallowing and voice effects of Lee Silverman Voice Treatment (LSVT ${ }^{\oplus}$ ): A pilot study. J Neurol Neurosurg Psychiatry 2002;71:31-6. 35.Sapir S, Ramig L, Fox C. Speech and swallowing disorders in Parkinson disease. Curr Opin Otolaryngol Head Neck Surg 2008;16:205-10.

http://dx.doi.org/10.1097/MOO.0b013e3282febd3a

36.Liotti M, Ramig LO, Vogel D, New P, Cook CI, Ingham RJ, et al. Hypo- 
phonia in Parkinson's disease: neural correlates of voice treatment revealed by PET. Neurology 2003;60:432-40.

37.Narayana S, Fox PT, Zhang W, Franklin C, Robin DA, Vogel D, et al. Neural correlates of efficacy of voice therapy in Parkinson's disease identified by performance-correlation analysis. Hum Brain Mapp 2010;31:222-36.

38.Dias AE. Reabilitação em grupo das disartrofonias em doentes de Parkinson pelo método Lee Silverman (Dissertação). São Paulo: Faculdade de Medicina da Universidade de Sáo Paulo, 2003, 100p.

39.Halpern A, Matos C, Ramig L, Petska J, Spielman J, Bennett J. LSVTC: A PDA supported speech treatment for Parkinson's disease. Presented at the Annual 9th International Congress of Parkinson's Disease and Movement Disorders, New Orleans, 2005;20(10): S134.

40.Kamińska, I, Zebryk-Stopa A, Pruszewicz A, Dziubalska-Kolaczyk K, Polczyńska-Fiszer M, Pietrala D, et al. The progress in the rehabilitation of dysarthria in Parkinson disease using LSVT (Lee Silverman Voice Treatment). Otolaryngol Pol 2007;61:713-8.

41.Spielman J, Ramig LO, Mahler L, Halpern A, Gavin WJ. Effects of an extended version of the lee silverman voice treatment on voice and speech in
Parkinson's disease. Am J Speech Lang Pathol 2007;16:95-107.

http://dx.doi.org/10.1044/1058-0360(2007/014)

42.Tindall R, Huebner RA, Stemple JC, Kleinert HL. Videophone-delivered voice therapy: a comparative analysis of outcomes to traditional delivery for adults with Parkinson's disease. Telemed J E Health 2008;14:1070-7.

http://dx.doi.org/10.1089/tmj.2008.0040

43. Howell S, Tripoliti E, Pring T. Delivering the Lee Silverman Voice Treatment (LSVT) by web camera: a feasibility study. Int J Lang Commun Disord 2009; 44:287-300.

http://dx.doi.org/10.1080/13682820802033968

44.Constantinescu GA, Theodoros DG, Russel TG, Ward EC, Wilson SJ, Wooton R. Home-based speech treatment for Parkinson's disease delivered remotely: a case report. J Telemed Telecare 2010;16:100-4.

http://dx.doi.org/10.1258/jtt.2009.090306

45.Dias AE, Limongi JCP, Barbosa ER. The Lee Silverman Voice Treatment method in the rehabilitation of hypophonia in Parkinson's disease: new perspective. Presented at the 14th International Congress of Parkinson's Disease and Movement Disorders, Buenos Aires, 2010;11:LB-29. 